\title{
Airy trajectory engineering in dynamic linear index potentials
}

\author{
Nikolaos K. Efremidis \\ Department of Applied Mathematics, University of Crete, 71409 Heraklion, \\ Crete, Greece (e-mail: nefrem@tem.uoc.gr)
}

Received May 31, 2011; accepted June 30, 2011; posted July 8, 2011 (Doc. ID 148529); published September 26, 2011

\begin{abstract}
We study the propagation of Airy beams in transversely linear index potentials with a gradient that is dynamically changing along the propagation direction. We find exact solutions in the case of Airy and apodized (Gaussian and exponentially) Airy beams in $1+1$ and $2+1$ dimensions. More important, we find that the Airy beam can follow any predefined path, in which case the potential gradient is determined as a function of this path. (c) 2011 Optical Society of America

OCIS codes: $\quad 050.1940,350.5500$.
\end{abstract}

The study of beams of the Airy type has attracted a lot of attention over the past few years because of its unique characteristics [1,2]. In particular, Airy beams have an asymmetric profile, are nondiffracting, and propagate along a self-bending parabolic trajectory. Because of these properties, Airy beams find potential applications in particle manipulation [3] , filament generation [ㄴ] optical bullet formation [ $[\underline{5}, \underline{6}]$, and abrupt autofocusing [-10].

One of the basic properties of the Airy beam is that it follows a parabolic trajectory as it propagates in homogeneous media. Specific generalizations of the Airy wave that are not diffraction-free can follow more complicated orbits, such as power law and hyperbolic $[10,11]$. Furthermore, an Airy beam propagating in a linear potential can follow any form of parabolic trajectory [12].

In this Letter, we study the dynamics of an Airy beam propagating in a transversely linear index potential, with an index gradient that is dynamically changing along the propagation direction. The problem is exactly solved using the method of characteristics in the Fourier space. We find diffraction-free Airy as well as Gaussian and exponentially apodized Airy beam solutions. In each case, the trajectory is determined from the $z$-dependent index gradient. The inverse problem is also solvable, according to which the potential is determined as a function of a predefined trajectory. We extend our results in $2+1 D$, in which case two-dimensional (2D) Airy beams can follow predesigned trajectories in space.

We start our analysis by considering the diffraction dynamics of an optical field $u$ as described by the paraxial approximation

$$
i u_{z}+\frac{1}{2} u_{x x}-\frac{d(z) x}{2} u=0,
$$

where $x$ is the scaled transverse coordinate, $z$ is the normalized propagation distance, and $d(z) x / 2$ is the transversely linear potential with a gradient $d(z)$. Experimentally, such an index structure can be generated by a wedged slab waveguide with an angle that varies along $z$. Furthermore, a $z$-dependent thermal gradient can induce an index gradient inside a slab waveguide. In plasmonics, such a potential might be generated in a wedged metal structure, where the one metal plate is tilted at an angle that varies along $z$ [12].

Equation (1) can be solved by utilizing the Fourier transform pair

$$
\tilde{u}=\int_{-\infty}^{\infty} u e^{-i k x} \mathrm{~d} x, \quad u=\frac{1}{2 \pi} \int_{-\infty}^{\infty} \tilde{u} e^{i k x} \mathrm{~d} k,
$$

in which case we derive a first-order partial differential equation:

$$
\tilde{u}_{z}-\frac{d(z)}{2} \tilde{u}_{k}=-i \frac{k^{2}}{2} \tilde{u} .
$$

The associated characteristic system,

$$
\frac{d z}{1}=\frac{d k}{-d(z) / 2}=\frac{d \tilde{u}}{-i k^{2} \tilde{u} / 2},
$$

can be solved by direct integration. In particular, equating the first and the second terms in Eq. (4) results in

$$
\kappa(z)=k-\frac{D(z)}{2},
$$

where

$$
D(z)=\int_{0}^{z} \mathrm{~d}(s) \mathrm{d} s .
$$

Equating the first and the third terms in Eq. (4), using Eq. (5), and then applying the inverse Fourier transform, we obtain

$$
u(x, z)=\frac{1}{2 \pi} \int_{\mathbb{R}} \tilde{u}(k, 0) e^{-\frac{i}{2} \int_{0}^{z} \kappa(s)^{2} \mathrm{~d} s} e^{i \kappa(z) x} \mathrm{~d} k .
$$

We focus our attention on Airy type initial conditions

$$
u(x, z=0)=\operatorname{Ai}\left(\gamma^{1 / 3} x\right),
$$

where $\gamma$ is the width of the Airy wave. By substituting the Fourier transform of Eq. (8),

$$
\tilde{u}(k, z=0)=\frac{1}{\gamma^{1 / 3}} \exp \left[\frac{i k^{3}}{3 \gamma}\right],
$$

into Eq. (7) and applying the transformation $k \rightarrow k+\gamma z / 2$ we obtain 


$$
u(x, z)=e^{\phi(x, z)} \operatorname{Ai}\left(\gamma^{1 / 3} \mu(x, z)\right),
$$

where

$$
\begin{gathered}
\mu(x, z)=\frac{F_{1}(z)}{2}+x-\frac{\gamma z^{2}}{4} \\
\phi=i \frac{\gamma F_{1}(z) z}{4}-i \frac{D(z) x}{2}-i \frac{F_{2}(z)}{8}+i \frac{\gamma z x}{2}-i \frac{\gamma^{2} z^{3}}{12}, \\
F_{1}(z)=\int_{0}^{z} D(s) \mathrm{d} s, \quad F_{2}(z)=\int_{0}^{z} D^{2}(s) \mathrm{d} s
\end{gathered}
$$

We consider that the Airy wave trajectory is given by the condition $\mu(x, z)=0$, i.e.,

$$
x=-\frac{F_{1}(z)}{2}+\frac{\gamma z^{2}}{4},
$$

that mainly determines its caustic [the maximum amplitude is determined via $\gamma^{1 / 3} \mu(x, z) \approx-1$ ]. If we expand the function $d(z)$ in a Taylor series as $d(z)=\sum_{j=0}^{\infty} c_{j} z^{j}$, then $F_{1}(z)=\sum_{j=0}^{\infty} c_{j} z^{j+2} /[(j+1)(j+2)]$ does not include the first two terms of a general Taylor expansion. We can account for these terms in the trajectory by considering that the initial condition is displaced by $x_{0}$ and tilted at an angle $\psi\left[u(x, z=0)=\operatorname{Ai}\left(\gamma^{1 / 3}\left(x-x_{0}\right)\right) e^{i \psi x}\right]$. The full formula is a trivial generalization of Eqs. (10)-(13) that is algebraically more involved and therefore is omitted. In particular, the phase of the beam is modified, and its trajectory now becomes

$$
x=x_{0}+\psi z-\frac{F_{1}(z)}{2}+\frac{\gamma z^{2}}{4} .
$$

More important, we can also solve the inverse problem: given a predefined Airy beam trajectory $x=g(z)$, we can determine the index gradient $d(z)$ as well as the initial condition parameters $x_{0}, \psi$ that are required. Following the relevant algebra we find that

$$
x_{0}=g(0), \quad \psi=g^{\prime}(0), \quad d(z)=\gamma-2 g^{\prime \prime}(z) .
$$

Apodized Airy beams have finite power and thus are experimentally realizable. Below we find exact expressions in the case of two different types of apodization. The first type of apodization is exponential, i.e., the initial condition is given by $u(x, z=0)=\operatorname{Ai}\left(\gamma^{1 / 3} x\right) e^{a x}$ [1]. The propagation of this latter beam is found by utilizing Eq. (7) and is given by

$$
u(x, z)=\operatorname{Ai}\left[\gamma^{1 / 3}\left(i a z+\frac{F_{1}}{2}+x-\frac{\gamma z^{2}}{4}\right)\right] e^{\phi(x, z)},
$$

where

$$
\begin{aligned}
\phi(x, z)= & \frac{i a^{2} z}{2}+\frac{a F_{1}}{2}+a x-\frac{a \gamma z^{2}}{2}-\frac{i x D}{2}+\frac{i z \gamma F_{1}}{4} \\
& -\frac{i F_{2}}{8}+\frac{i \gamma x z}{2}-\frac{i \gamma^{2} z^{3}}{12}
\end{aligned}
$$

and $F_{1}, F_{2}$ are given by Eqs. (13). Effectively, the imaginary part in the argument of the Airy function does not modify the trajectory that the caustic of the Airy beam follows [Eqs. (14) and (15)], at least up to the point where the beam does not exhibit significant diffraction.

Using the Fourier integral (7) we can also find exact solutions for Gaussian apodized Airy beams [13]. Inparticular, assuming an initial condition $u(x, z=0)=$ $\operatorname{Ai}\left(\gamma^{1 / 3}\left(x-c_{0}\right)\right) e^{-(x / w)^{2}}$, where $c_{0}$ is the relative displacement between the Airy and the Gauss and $w$ is the initial width of the Gaussian, we find that

$$
u(x, z)=\frac{e^{\phi}}{\sigma^{1 / 2}} \operatorname{Ai}\left[\gamma^{1 / 3}\left(\frac{1}{\sigma}\left(x+\frac{F_{1}}{2}\right)-c_{0}-\frac{\gamma z^{2}}{4 \sigma^{2}}\right)\right],
$$

where

$$
\begin{aligned}
\phi(x, z)= & \frac{-1}{\sigma w^{2}}\left(x+\frac{F_{1}}{2}\right)^{2}-\frac{i F_{2}}{8}-\frac{i x D}{2} \\
& +\frac{i z}{2 \sigma}\left[\frac{\gamma}{\sigma}\left(x+\frac{F_{1}}{2}\right)-\gamma c_{0}-\frac{\gamma^{2} z^{2}}{6 \sigma^{2}}\right] .
\end{aligned}
$$

$\sigma=1+\left(2 i z / w^{2}\right)$, and $F_{1}, F_{2}$ are given by Eqs. (13). As can be seen from the argument of the Airy function, the trajectory of the Airy beam is modified due to the presence of the Gaussian.

Typical examples of Airy beam engineering include sinusoidal, power law, exponential and logarithmic, to mention a few. In all of the cases presented below, we use exponentially apodized Airy beams with $a=0.05$. Let us first consider the power law trajectory

$$
x=g(z)=c\left(z-z_{0}\right)^{b} .
$$

Solving Eqs. (16) we find that the initial displacement and tilt of the Airy beam are given by $x_{0}=c\left(-z_{0}\right)^{b}$, $\psi=b c\left(-z_{0}\right)^{b-1}$ while the potential gradient function satisfies $d(z)=1-2(b-1) b c\left(z-z_{0}\right)^{b-2}$ provided that
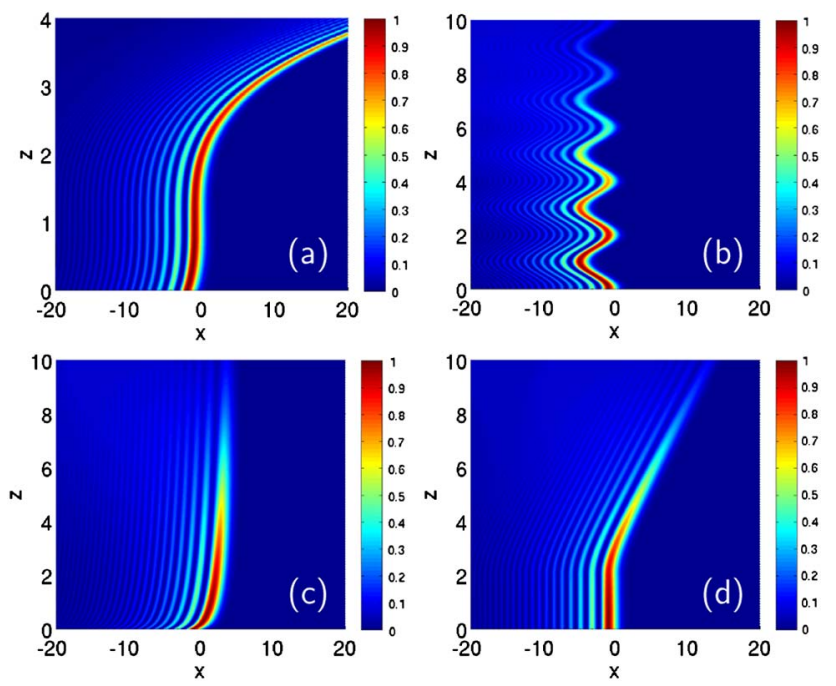

Fig. 1. (Color online) Exponentially apodized Airy beam propagation $(a=0.05)$ along predefined paths. (a) Power law trajectory $x=(z-1)^{3}$, (b) sinusoidal trajectory given by Eq. (22), (c) logarithmic trajectory $x=\log (1+10 z)$, (d) hyperbolic trajectory given by Eq. (23). 


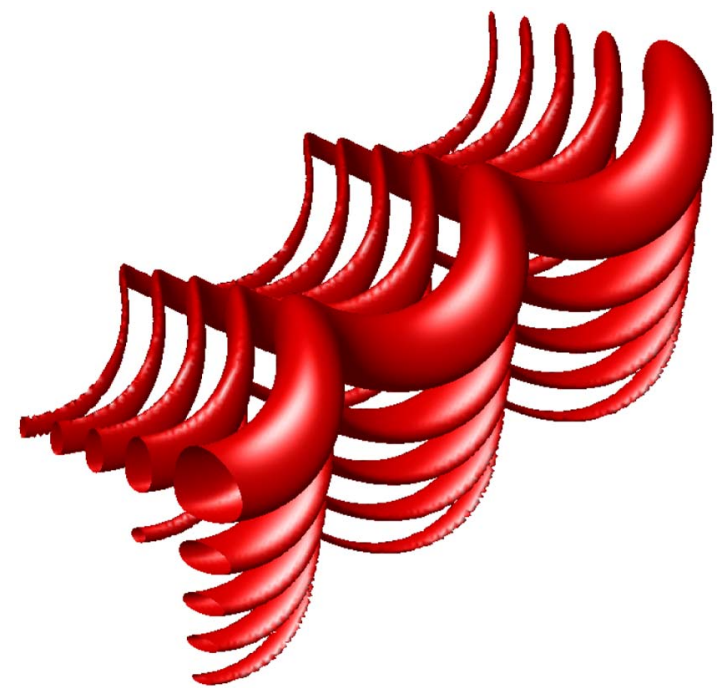

Fig. 2. (Color online) Schematic illustration of a 2D Airy beam following a spiral trajectory in space.

the previous expressions are real. In Fig. 1(a) we see a typical power law trajectory of an Airy beam in the case where $c=1, b=3, z_{0}=1$. In Fig. $\underline{1(b)}$, an Airy beam propagates according to the periodic trajectory

$$
x=g(z)=2(\cos (\pi z)-1) .
$$

Solving Eqs. (16), we obtain $d(z)=1+4 \pi^{2} \cos (\pi z)$, along with $x_{0}=\psi=0$. In Fig. 1(c), the Airy beam follows the logarithmic trajectory $x=\log (1+10 z)$.

Note that there are some physical limitations in the possible trajectories that the Airy beam can follow. In particular, a discontinuous trajectory $x=g(z)$ is related to a gradient $d(z)$ that involves $\delta^{\prime}$ functions. A continuous $g(z)$ with a discontinuous first derivative $g^{\prime}(z)$ requires Dirac $\delta$ functions in $d(z)$. If the second derivative $g^{\prime \prime}(\boldsymbol{z})$ is discontinuous, then $d(z)$ should also be discontinuous. Finally, if $g(z), g^{\prime}(z)$, and $g^{\prime \prime}(z)$ are continuous, then the index gradient is also a continuous function.

As an example of an Airy beam propagating in a medium with an abrupt change in the gradient, we consider the following trajectory with a discontinuous second derivative:

$$
x=g(\boldsymbol{z})=2\left(\sqrt{(\boldsymbol{z}-2)^{2}+1}-1\right) H(z-2),
$$

where $H(x)$ is the Heaviside step function. For $z<2$ the Airy beam follows a straight path, whereas for $z>2$ the beam bends and follows a hyperbolic path with an asymptotic $x=2(z-2)$ [see Fig. 1(d)]. In this case, the required index gradient is discontinuous and is given by

$$
d(\boldsymbol{z})=1-\frac{4 H(z-2)}{((z-4) z+5)^{3 / 2}} .
$$

The above results can be generalized in the case of two transverse dimensions. In particular, the normalized paraxial equation in $2+1 D$ reads

$$
i u_{z}+\frac{1}{2} u_{x x}+u_{y y}-\frac{1}{2}\left[d_{1}(z) x+d_{2}(z) y\right] u=0,
$$

where the linear index gradient along $x(y)$ is modulated along $z$ as $d_{1}(z)\left[d_{2}(z)\right]$. Applying separation of variables

$$
u(x, y, z)=v(x, z) w(y, z),
$$

we find that each component satisfies

$2 i v_{z}+v_{x x}-d_{1}(z) x v=0, \quad 2 i w_{z}+w_{y y}-d_{2}(z) y w=0$.

The initial condition is a 2D Airy wave of the form

$$
u(x, y, 0)=\operatorname{Ai}\left[\gamma^{1 / 3}\left(x-x_{0}\right)\right] \operatorname{Ai}\left[\gamma^{1 / 3}\left(y-y_{0}\right)\right] e^{i \psi_{1} x+i \psi_{2} y},
$$

where $\left(x_{0}, y_{0}\right)$ is the initial displacement and $\left(\psi_{1}, \psi_{2}\right)$ is the initial tilt of the beam along $(x, y)$, respectively. The values of $d_{1}(\boldsymbol{z}), d_{2}(\boldsymbol{z}), x_{0}, y_{0}, \psi_{1}, \psi_{2}$ can now be engineered so that the Airy beam will follow the predefined trajectory $x=g_{1}(z), y=g_{2}(z)$. More specifically, we find that $x_{0}=g_{1}(0), y_{0}=g_{2}(0), \psi_{1}=g_{1}^{\prime}(0), \psi_{2}=$ $g_{2}^{\prime}(0), d_{1}(\boldsymbol{z})=\gamma_{1}-2 g_{1}^{\prime \prime}(\boldsymbol{z}), d_{2}(\boldsymbol{z})=\gamma_{2}-2 g_{2}^{\prime \prime}(\boldsymbol{z})$. As an example, in Fig. 2 we can see a schematic illustration of a 2D Airy beam propagating along the spiral trajectory $x=\cos (z)-1, y=\sin (z)$.

In conclusion, we have studied the propagation of Airy beams in transversely linear index potentials with a gradient that is dynamically changing along the propagation direction. Both $1+1 D$ and $2+1 D$ configurations have been studied. Exact solution of Airy as well as Gaussian and exponential apodized Airy beam are derived. We have shown that by engineering the index gradient the Airy beam can follow any predefined trajectory.

\section{References}

1. G. A. Siviloglou and D. N. Christodoulides, Opt. Lett. 32, 979 (2007).

2. G. A. Siviloglou, J. Broky, A. Dogariu, and D. N. Christodoulides, Phys. Rev. Lett. 99, 213901 (2007).

3. J. Baumgartl, M. Mazilu, and K. Dholakia, Nat. Photon. 2, 675 (2008).

4. P. Polynkin, M. Kolesik, J. V. Moloney, G. A. Siviloglou, and D. N. Christodoulides, Science 324, 229 (2009).

5. A. Chong, W. H. Renninger, D. N. Christodoulides, and F. W. Wise, Nat. Photonics 4, 103 (2010).

6. D. Abdollahpour, S. Suntsov, D. G. Papazoglou, and S. Tzortzakis, Phys. Rev. Lett. 105, 253901 (2010).

7. N. K. Efremidis and D. N. Christodoulides, Opt. Lett. 35, 4045 (2010).

8. D. G. Papazoglou, N. K. Efremidis, D. N. Christodoulides, and S. Tzortzakis, Opt. Lett. 36, 1842 (2011).

9. P. Zhang, J. Prakash, Z. Zhang, M. S. Mills, N. K. Efremidis, D. N. Christodoulides, and Z. Chen, Opt. Lett 36, 2883 (2011).

10. I. Chremmos, N. K. Efremidis, and D. N. Christodoulides, Opt. Lett. 36, 1890 (2011).

11. E. Greenfield, M. Segev, W. Walasik, and O. Raz, Phys. Rev. Lett. 106, 213902 (2011).

12. W. Liu, D. N. Neshev, I. V. Shadrivov, A. E. Miroshnichenko, and Y. S. Kivshar, Opt. Lett. 36, 1164 (2011).

13. M. A. Bandres and J. C. Gutiérrez-Vega, Opt. Express 15, 16719 (2007). 